\title{
Outreach for Science Facilities: Armagh Observatory and Planetarium's programme for the Cherenkov Telescope Array
}

\section{Michael G. Burton, ${ }^{*}$ Kerem Osman Cubuk and Heather Alexander}

Armagh Observatory and Planetarium,

College Hill, Armagh, Northern Ireland, UK

E-mail: michael.burton@armagh.ac.uk, kerem.cubuk@armagh.ac.uk, heather.alexander@armagh.ac .uk

We describe an outreach programme being delivered by the Armagh Observatory and Planetarium (AOP) for the Cherenkov Telescope Array (CTA). Founded in 1790 and with a rich astronomical heritage, AOP today combines the research and education arms of our organisation to bring a research-informed outreach programme to the public, most often through our planetariumrelated activities. We have developed and written, in-house, a short full dome planetarium show ("Exploring the High-Energy Universe") that describes the science of gamma-ray astronomy and introduces the CTA as the first ground-based gamma-ray observatory open to the whole scientific community. In parallel, we are engaged in developing a series of short videos to introduce the scientists and the science of the UK CTA consortium, again designed for public audiences. These videos can be accessed through our social media channels. Delivery of such outreach programme in bite-sized pieces is an essential element in attracting and engaging audiences. We explain how we have developed the skill set to do this in our Education Team at AOP whilst our facility has been closed for the past year, a result of the Covid-pandemic. There is also scope in extending these concepts for providing outreach support for other science facilities.

$37^{\text {th }}$ International Cosmic Ray Conference (ICRC 2021)

July 12th - 23rd, 2021

Online - Berlin, Germany

\footnotetext{
*Presenter
} 


\section{The Armagh Observatory and Planetarium}

The Armagh Observatory and Planetarium (AOP) contains one of the world's oldest observatories and planetariums. Founded in 1790, the Observatory has been in continuous use for scientific research ever since. Six generations of telescopes are found within, still in situ, largely as they were when used for pioneering science in their day. Key innovations in the development of the modern clock-driven equatorial telescope through the $18^{\text {th }}$ and $19^{\text {th }}$ centuries can be experienced in a tour of the Observatory. Today, these telescopes sit alongside the research offices of the current astronomers, in what were formerly the living quarters of past Directors and their families. The astronomers, however, are now conducting their research using the range of international facilities available to the $21^{\text {st }}$ century scientific community, and not those on site in Armagh.

The Armagh Planetarium was opened in 1968, and today is the oldest operating planetarium in the British Isles. Only a handful of planetaria elsewhere in the world are older. In turn, it also has a significant history in its field having developed a reputation for innovation within the planetarium industry, for instance in the introduction of video technology into dome shows. Today, it attracts around 50,000 visitors per year (pre-Covid), a remarkably high number given that the population of the City of Armagh is just $\sim 15,000$ people.

The two institutions, the Armagh Observatory and the Armagh Planetarium, while on the same site nevertheless operated independently until their merger in 2016. The intent of the merger was to unlock the synergies that might be obtained between the research and the education \& outreach arms of the new organisation - the AOP. Below we describe one of the products of that merger research-informed outreach programmes.

\subsection{A Capacity for Research-informed Outreach}

It is unusual to have both a scientific research organisation and an outreach organisation on the same site, certainly one with such a rich scientific heritage. This has now allowed a close relationship between the activities of each to be developed. Instead of the traditional star show, illustrating the the constellations and their mythology, which typically dominates presenter-led shows at planetaria, we have been able to enrich our offerings by including elements of current research and the scientific pedigree of the Observatory within them.

Researchers are, of course, leaders in their fields, but can have difficulty encapsulating the messages of their work into the bite-sized chunks needed for public presentation. Our education team are skilled in presenting messages to the public in the short, accessible and entertaining style which is needed, not overwhelming them with all the facts and caveats that research scientists generally also want to explain. However, they need to be guided as to what the key science is, and in interpreting it while simplifying the content and ensuring the essential message remains, and is, most importantly, not wrong.

Developing such synergies between the two arms of our organisation has not happened overnight, it has not been easy, and indeed is not complete. This will be a continuous and ongoing process, one we need to always work at to ensure that it remains embedded in the culture of AOP. Nevertheless, in the five years it has now been since the merger, we are proud to say that we are achieving success in delivering research-informed public outreach in science. 
This will be immediately evident to any visitor to the new exhibition hall in the Armagh Planetarium, which now incorporates elements from the scientific heritage of the Observatory and current research activity, within the displays about planets, stars and galaxies that are also typically found in any astronomically-focussed science visitor centre.

\section{The Planetarium Dome}

We are incorporating similar elements within our presentations under the planetarium dome, for instance the walk-in sequence as visitors wait for the show to begin now illustrates the heritage telescopes just a short walk away in the Observatory. Scenes in the dome shows now include elements of current research taking place in Armagh. All these are delivered in bite-sized chunks, as it needs to be for a public show, as a part of the immersive experience under the dome of a planetarium.

The essential element of a full dome planetarium is the shared experience of the audience. The presenter is taking you, the viewer, on a tour of the cosmos, along with your companions, a tour which can go literally anywhere in space and time (with the obvious proviso that the planetarium software needs to have celestial objects programmed within it that are available for that epoch and 3D location in space). This experience is shared with everyone else sitting under the dome. This is what differentiates it from the VR experience, which, while also immersive, is for a journey that you are taking alone.

A modern digital planetarium is essentially a sophisticated exercise in scientific data visualisation. The components of cosmos, whether they be from the solar system, stars or galaxies whose positions and parameters are held in the projector system's database, are rendered in real time onto the dome surface, as seen from any location the presenter chooses to take you to. Journeys between places and/or epochs can be simulated (generally at speeds $>>c$ !), realistic colourings and shadings applied, with surround-sound audio added, to create a dynamic, real-time and inspiring visualisation. It is the power of the planetarium's graphic processors that enable the real-time rendering that makes such presentations now possible. Pre-rendered dome shows can also be shown, though this is more akin to a movie-experience with the giant screen, as they always follow the same sequence of events. However, in a presenter-led show that experience can be altered each time, dependent on the interests and inclinations of both presenter and audience alike.

\subsection{Developing a Dome Show}

The presenter generally sits in front of a terminal to deliver their shows in a planetarium, picking from a set of (virtual) buttons to take the viewer to pre-defined destinations (e.g. the planets), with a date \& time controller to set the epoch and duration of the journey, and other controllers to change the viewing angle, orientation and illumination. These buttons are then often simplified by being placed together in a single page control panel, so the presenter simply pushes each button in turn to go on the journey, narrating the experience to the audience while doing so.

However, it also possible to define these actions through a scripting language, basically a set of commands that set the objects to be viewed on the dome and occur in a set sequence and with set times between them. This might, for instance, start the show by showing the location of the host planetarium seen from above, take-off and orbit the Earth, and then go on a journey to a series of 
celestial objects. The objects themselves may be represented by the best imagery available, or in some cases 3D renderings based on the interpretation of scientific data which allows the viewer to experience how the vista might look from a hypothetical space craft at the celestial location. To the surface of the dome it is also possible to add text, imagery and video, as well as bring in suitable audio, so adding to the experience and amplifying the story.

A sequence of scripts can then be run sequentially to produce a dome show. While a fully produced full dome show rendered into a suitable video format for sale by a vendor might cost 6or 7-figure sums to produce, and be sold to planetaria for 4- or 5-figure amounts, producing a dome show through a series of scripts is actually free, aside of course from the time of the people who put it together. Further, cloud technology incorporated within the systems of major planetarium suppliers allows the scripts to be freely shared with other planetaria running the same software system, so shows can readily be played in many planetaria through the world at no additional cost to either the producer or planetarium. The experience might not match that of the fully professional production of a pre-rendered full dome show, but such is the power of the modern digital planetarium that the experience is still a powerful one, as well as being educational. Importantly, it provides an opportunity to produce planetarium shows that are within the capabilities of research scientists who can then use the dome to tell the stories of their own science.

At AOP we are now trying to do just this. Our first production was to tell the story of Armagh's weather record, the 225+ year continuous series of daily meteorological measurements we have conducted since 1795, on the occasion of being recognised by the World Meteorological Organisation as a Centennial Weather Station.

Our second production is aimed at contributing to the outreach programme for a project we recently joined as a member of the UK consortium in the Cherenkov Telescope Array (CTA) observatory. This is the next-generation gamma-ray telescope that is now being built in La Palma in the Canary Islands of Spain and on the Atacama plateau of Chile near to ESO's Paranal Observatory.

\section{A Dome Show for CTA: Exploring the High-Energy Universe}

We have written this show in five scenes, for a total show length of $\sim 10$ minutes. Specifically, of the three authors to this paper, Burton wrote the script and story board, Cubuk produced the show by scripting using the Digistar planetarium language, and Alexander recorded the audio.

The show begins by looking at the multi-wavelength Milky Way and the different phenomena that dominate our Galaxy's appearance as we change from optical, to infrared, to radio. Finally, we turn to the gamma-ray sky.

Having introduced the high-energy universe, the next two scenes travel from Earth to cosmic sources of gamma rays, first to the pulsar in the heart of the Crab Nebula, and then to the super massive black hole in the centre of M87. We start the journey with the view of the stars in the sky towards the relevant constellations, and then travel away from Earth, past the stars in our Galaxy to the Crab, and then past the galaxies in our Local Group to the Virgo cluster with M87 in its heart. Video and images are added to the dome to illustrate the phenomena of pulsars and winds from their jets, as well as black holes, accretion disks and their relativistic outflows.

The next scene returns to Earth to look at the kinds of telescopes astronomers are now using to explore the cosmos, first the more familiar optical and radio telescopes on the ground and Hubble 


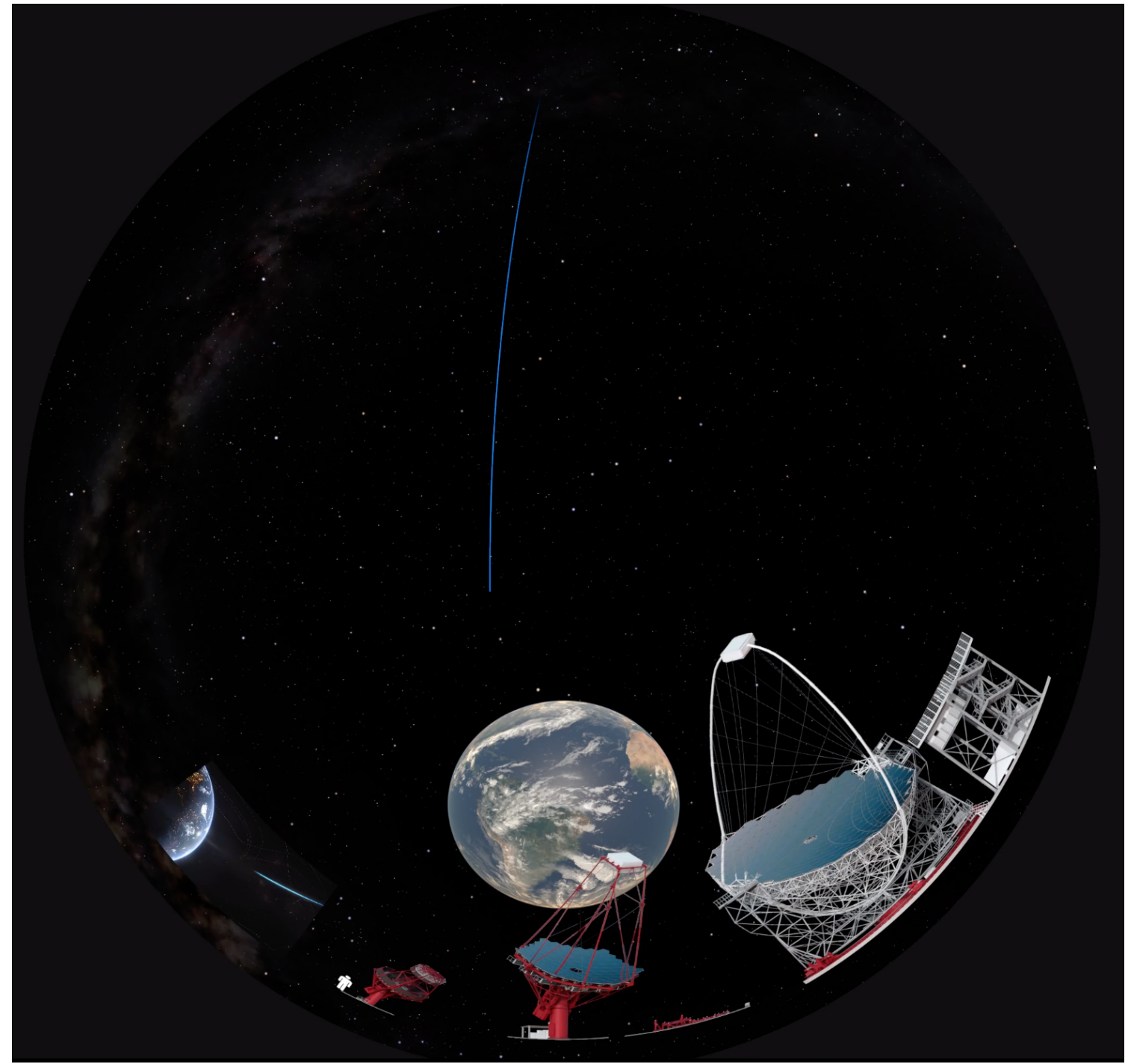

Figure 1: A screen grab from the CTA planetarium dome show, here showing the three different types of telescope being used by CTA, as well as the passage of a gamma ray towards Earth. The image from the hemispherical dome has been projected onto a flat screen, hence the circular field of view. The audience view is directed $30^{\circ}$ above horizontal, i.e., at the Earth in this image. The centre of screen is overhead in the planetarium dome and the top half of the image would be behind the viewer.

in space, before introducing gamma-ray telescopes through H.E.S.S. in Namibia and the concept of Cherenkov radiation. In this scene we view these telescopes from orbit, moving around the Earth accordingly.

The final scene then comes to the CTA, introduces the two sites in La Palma and Chile and shows how the southern site will look. It explains the three different types of optical telescope that are used to capture the Cherenkov flashes and why they are needed (i.e. related to measuring the gamma-ray spectrum as well as improving the image fidelity). Finally, we follow a gamma ray emitted from a cosmic source, travelling through space to Earth and inducing a Cherenkov cascade in the atmosphere when it arrives, and the resulting blue flash that will, so very briefly, envelope the 


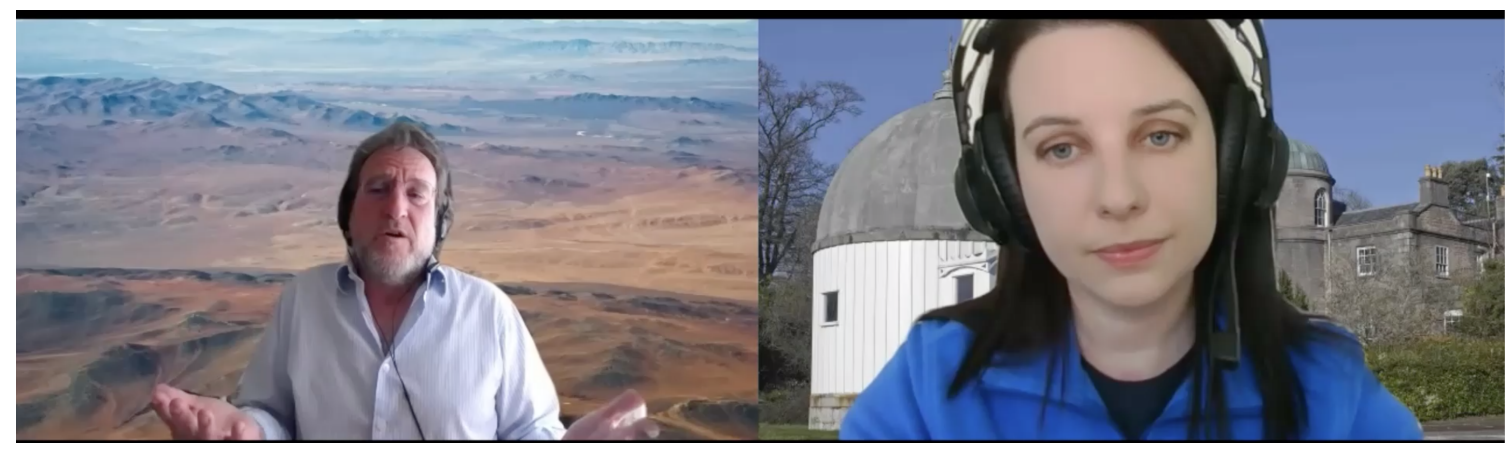

Figure 2: Jon Lapington, leader of the UK CTA consortium, being interviewed by Heather Alexander for the "Get to Know You" series introducing the scientists of the project.

telescopes. At the same time, we also show alongside on the dome extracts from a video that the CTA project has produced, illustrating these concepts further as well as how the data are recorded and processed. We end by puzzling about the enigma of the most extreme phenomena in nature that scientists will be able to explore using the CTA.

The dome show has been written and produced entirely in-house at Armagh, but as of the time of writing has yet to be shown even in our planetarium in Armagh due to the Covid lockdown. All we have been able to do is share it with audiences via screen share of a flat screen version over Zoom (see Fig. 1), which of course cannot provide the full immersive experience of the Dome. Once we are able to launch the show in the Armagh Planetarium then it will be made freely and publicly available through the Digistar cloud to other planetaria around the world. It may also be rendered into other formats for showing in planetaria with different projector systems.

\section{Get to Know the Scientists Interviews}

Another contribution AOP are making to the CTA's outreach programme is through a series of short get-to-know-you interviews with scientists involved in the project, in particular those within the UK CTA consortium. During the Covid lock down our Planetarium in Armagh has, of course, been closed to the public. Our education team, used to delivering in-person activities to our visitors, have had to re-invent themselves in order to continue delivering education and outreach programmes. We came up with the concept of "Science at Home", short videos demonstrating easy-to-make experiments you could try at home. This developed into a whole range of online activities using our social media channels ${ }^{1}$. Blog articles about the sky and about the science ${ }^{2}$, interviews with our scientists, short talks on astronomy (Astrobytes), Star Trackers - live star shows (using Stellarium) with robotic telescope viewing (using Slooh) and audience interaction using Zoom aimed at family audiences, Cosmic Classroom (a similar programme but targeted towards schools), and a podcast series entitled "Intergalactic Craic" 3 and now in its second series.

\footnotetext{
${ }^{1}$ See facebook.com/armaghplanet, youtube.com/user/ArmaghPlanetarium, twitter.com/armaghplanet, instagram.com/armaghplanet

${ }^{2}$ See armaghplanet.com

${ }^{3}$ See anchor.fm/intergalacticcraic
} 
A particular success was the " 5 Questions" interview series, where the team approached prominent scientists and asked if they'd like to be interviewed, nominally through five questions. One example is an interview with Helen Sharman, the first British astronaut, the full series can be accessed via AOP's YouTube channel. We have now adapted this programme to tell the stories behind the scientists involved in the the collaborations we work with, and in particular the CTA. An immense project, with over 1,000 scientists contributing towards its future completion, bringing with them a comprehensive range of skill sets to the table. In such a project, while the headline science aims can be prominent, the essential contributions coming from the enabling disciplines that make this all possible can get lost, to the public as well as to the funding bodies.

We (Alexander) have now recorded the first interview in this new initiative, with Professor Jon Lapington, leader of the UK consortium within CTA (see Fig. 2). His team are responsible for building some of the cameras and associated technologies for the small-sized telescopes (the $4 \mathrm{~m}$ diameters SSTs). Through this interview programme we aim to bring you the scientists behind the science, and showcase the remarkable contributions they are making towards the success of this global project.

We also hope to be able expand these initiatives to providing outreach support for other science facilities in the future.

\section{Acknowledgments}

The Armagh Observatory and Planetarium (see www.armagh.space) is an Arms Length Body of the Department for Communities (DfC) in the Northern Ireland Executive. We are grateful to DfC for their grant support to our organisation that enables AOP's research, education and outreach programmes. The UK CTA consortium is supported by the Science and Technology Facilities Council (STFC). The audio for the Planetarium Dome show was recorded with the help of the Southern Regional College in Armagh.

This work was conducted in the context of the CTA Communications and Outreach group. We gratefully acknowledge financial support from the agencies and organisations for the CTAC that are listed here: http://www.cta-observatory.org/consortium_acknowledgments/. 